\title{
Use of Silicone Elastomer-Based Microfluidic Devices and Systems in Reproductive Technologies
}

\author{
Koji Matsuura and Keiji Naruse
}

Additional information is available at the end of the chapter

http://dx.doi.org/10.5772/47731

\section{Introduction}

Reproductive technologies (RT) play important roles in the conservation of biodiversity, the production of domestic animals such as pigs and cows, and in human infertility treatments [1-3]. Artificial insemination (AI) is a very useful tool to introduce superior genes with a minimal risk of disease as compared with natural mating [4]. Improvements in cryopreservation of semen as well as storage in its liquid form have made AI more accessible [5, 6]. In vitro manipulation of gametes for the production of embryos was first successfully applied in mice in 1958 and in rabbits in 1959 [7]. In initial experiments, matured oocytes retrieved from a female were fertilized with sperm in the laboratory and then transferred to a recipient mother. In 1981, the first live calf was produced by in vitro fertilization (IVF). Currently, the development and refinement of AI and IVF are concentrating on gamete and embryo collection, sorting and preservation of sperm, gametes, and embryos, and in vitro production of embryos, culture, and embryo transfer. These developments are facilitated by modern equipment for ultrasonography, microscopy, cryopreservation, endoscopy, and flow cytometry, as well as micromanipulation and centrifugation [1]. Microfluidic technologies have also contributed to recent developments in RT, especially over the past decade.

Microfluidic devices are powerful tools for handling reproductive cells, because the sizes of these cells are in the micrometer range. The length of a mammalian sperm is $50 \mu \mathrm{m}$, and the diameter of the mammalian oocyte and early embryo is approximately $100 \mu \mathrm{m}$; these are similar in all mammalian species. For cellular-level observations, transparent materials must be used. More than 10 years ago, microdevices for manipulation of reproductive cells were hand-made and sometimes reproducing these devices was laborious work. For example, to settle embryos in microwells, heated polished steel rods were pressed by hand to the bottom 
of the well of a polystyrene dish to prepare the microwells [8]. The current most popular technology for the fabrication of microfluidic devices for cell biological application is based on the soft-lithography of poly-dimethylsiloxane (PDMS). PDMS is a silicone elastomer, it is transparent, not detrimental to cells, possesses high permeability to gas, and is easy to fabricate; therefore, fabricated devices can be used for observing cells. Through simple molding procedures, the material can be made into microfluidic devices in short periods of time (typically, $<1$ day), and multiple copies of a device can be generated in several hours [9]. Its widespread use as a material of choice is because of its mechanical properties, which are amenable to integration of fluidic valves, essential elements for major microfluidic applications [10]. For biological use, reported applications of PDMS microfluidic devices are analyzing cells [11, 12], applying forces [13, 14], substrate patterning [15, 16], and creating chemical microenvironments [17-19]. Because of laminar flow in microfluids, experiments using these microfluidic devices for chemical gradient production and cellular manipulations are easily reproduced. These elastomer technologies and applications can be transferred to the development of RT.

This chapter introduces the usability of silicone elastomer-based microfluidic devices in RT. Since most readers are not likely to be familiar with RT, we will cover the following points: (1) the background to the use of elastomers in RT, (2) details of current research on sorting and analyzing motile sperm, (3) silicone elastomer-based microfluidic devices for creating static and dynamic mammalian embryo culture systems that can mimic the motion of the oviduct, and (4) the conclusion. This chapter introduces the current research areas for developing improvements in RT and suggests the possibility of using elastomers in human ART.

\subsection{Background to the use of elastomers in reproductive technologies}

RT refers to procedures that include in vitro handling of cattle or human oocytes, sperm, or embryos to induce pregnancy [20,21]. To improve fertilization rate in pigs and cattle, AI using washed sperm is the most widely used technique. Because the outcome of AI largely depends on the quality of semen and the insemination procedure, sperm washing and sperm motility analyses are part of conventional AI procedures [4]. Sperm washing by differential gradient centrifugation and swim-up of motile sperm can reduce the amount of virus present in the sample [22]. However, harmful effects of washing and centrifugation have been attributed to the generation of reactive oxygen species, which can irreversibly damage the sperm and impair their fertilizing ability [23-25]. This damage may be severe for cryopreserved sperm that have already been injured during freezing [25]. Some sperm motility analyses provide reliable estimation of the fertilizing ability of mammalian sperm such as computer-assisted sperm analysis (CASA) [26, 27]. A significant positive correlation with fertility was found for the linear-motility parameter [28]. Furthermore, the generally reduced fertility of cryopreserved semen is considered to be due to the short life-span of frozen-thawed sperms [29, 30]. Before AI, the likelihood of successful fertilization can be predicted by motility analyses.

The most common procedure performed to assist reproduction is in vitro fertilizationembryo transfer (IVF-ET). Conventional IVF treatment requires that the ovaries be 
stimulated with gonadotropins, which contain follicle-stimulating hormone (FSH) and luteinizing hormone ( $\mathrm{LH})$ in order to increase the number of mature oocytes retrieved, and thus the number of embryos available for transfer [31]. After washing the sperm to be used, the sperm-containing medium is dispensed in microdrops under paraffin oil or more simply in wells without oil [32]. At the same time, matured oocytes are introduced into the fertilization medium and co-incubated with sperm for $18-24 \mathrm{~h}$ at $37-39^{\circ} \mathrm{C}$ in $5 \% \mathrm{CO}_{2}$. The fertilization rate can be assessed indirectly by examination of the cleavage rate $40-42 \mathrm{~h}$ post insemination. The basic procedures of IVF in cattle and humans are similar [33]. Another in vitro procedure is intracytoplasmic sperm injection (ICSI), where a selected single sperm is directly injected into the oocyte. For the ICSI procedure, the oocyte is immobilized using a holding pipette; an injection pipette with an internal diameter of $6 \mu \mathrm{m}$ is used to aspirate a single spermatozoon. Before aspiration, the sperm is immobilized in polyvinylpyrrolidone. A morphologically normal sperm is aspirated into the injection needle, tail first. Immobilization of the sperm can also be achieved by crushing the tail with the injection pipette. The injection pipette is passed through the zona pellucida and the membrane of the oocyte into the cytoplasm in a position sufficiently distant from the first polar body. After ICSI using ejaculated sperm, more than two-thirds of the injected oocytes became normally fertilized [34]. This procedure is prominent in assisted human reproduction. ICSI is feasible in cattle, even with freeze-dried sperm; however, it is not yet widely applied [20,35].

The fertilized embryos are cultured in microdrops under paraffin oil. First cleavage occurs 23-29 $\mathrm{h}$ after fertilization, and the cleaved embryo is called a 2-cell embryo [36]. The number of blastomeres in an embryo increases to 4 and 8 at the second and third cleavage, which occur at days 2 and 3, respectively. Compaction of the cleaving embryo begins on day 3 due to formation of tight inter-cellular junctions. The embryos secrete factors that sustain their development, and for this reason they grow better in groups than alone [37]. During blastocyst formation, two clearly distinguishable cell lines are formed, the inner cell mass (ICM) and the trophectoderm (TE) [36]. A full human blastocyst at day 5 of development should consist of more than 60 cells and should at least double its cell number on day 6 [36]. Embryos of excellent and good quality at the compact morula to blastocyst developmental stages yield the highest pregnancy rates [38]. Recently, non-surgical embryo transfer techniques involving the use of specialized embryo transfer pipettes have been developed [38]. Because the most important factor influencing the rate of multiple births is the number of embryos transferred, in human assisted RT (ART), single embryo transfer is considered to be appropriate $[39,40]$. IVF-ET is the most important process in animal and human RT. The scheme of human ART is summarized in Figure 1.

Sperm washing and selection are important processes for improving the fertilization rates in IVF and ICSI, and these procedures are the same as those used in AI. The quality of the embryo formed in in vitro embryo culture is related to the fertilization rate. Therefore, these processes need to be studied further in order to improve the success rate of RT. In vitro culture systems can and must be improved to obtain embryos of normal quality, equal to those obtained by development in the oviduct. A better understanding of the many factors regulating embryonic development and interaction with the female reproductive tract might 
provide some insights [41]. In conventional IVF-ET procedures, sperm are separated by nonphysiological centrifugation and embryos are cultured under static conditions. The far from physiological conditions of these methods could be one of the reasons for the reduced pregnancy rate following the use of RT. Researchers in this field have to reconsider these current methods in order to further improve the success rate of RTs.

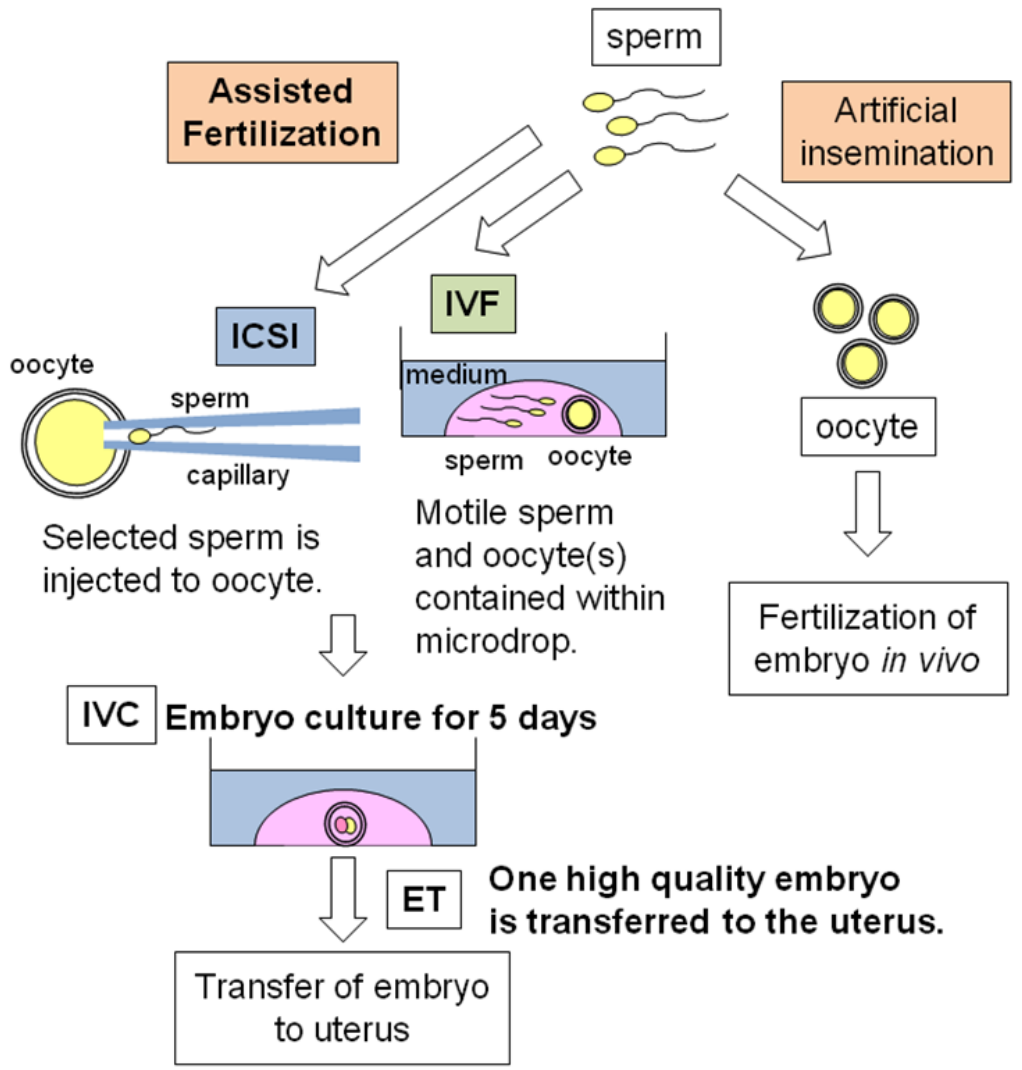

Figure 1. Schematic representation of human ART

The mammalian oviduct and uterus under physiological conditions have been studied to aid the design of microfluidic systems for RT. The oviduct consists of three segments, each with different functions: the uterotubal junction, the isthmus, and the ampulla [42]. The uterotubal junction provides a barrier to infectious microbes that might enter the oviduct from the uterus. The isthmus serves as a sperm storage organ and the ampulla provides an environment conducive to fertilization and early embryonic development. Figures $2 \mathrm{~A}$ and $\mathrm{B}$ show the dynamic structural changes in the diameter of the oviduct induced by the peristaltic movement of the rat oviduct stained with fluorescent dyes. The stained microstructure in the isthmus has moved. The embryos' motion is caused by this peristaltic movement, and non-motile sperm are washed out from the oviduct. 

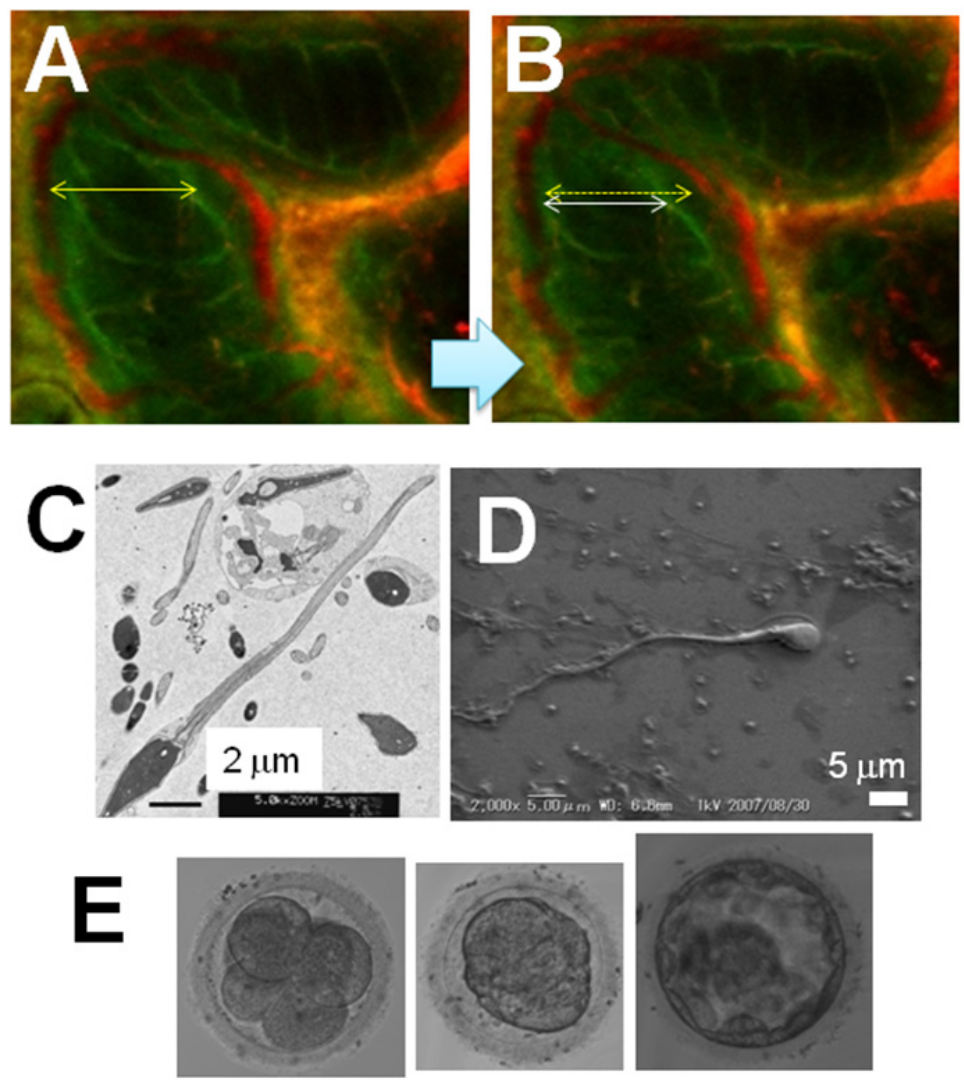

Figure 2. (A) and (B) The rat oviductal structure. Fluorescence image (B) was recorded $10 \mathrm{~s}$ after recording (A). The yellow arrow in (A) and the yellow dotted arrow in (B) are of the same length. The distance of the inner wall of the oviduct decreased in (B) due to peristaltic movements. (C) TEM and (D) SEM images of mammalian sperm. (E) Human embryos: (left) Cleaving embryo, (center) Morula, (right) Blastocyst.

Figures $2 \mathrm{C}$ and $\mathrm{D}$ show electron microscopic images of human sperms, indicating that the length is approximately $50 \mu \mathrm{m}$. The sperm head contains the DNA, the midpiece contains mitochondria, and the tail assists with swimming towards the oocyte [43]. Ejaculated sperm in the oviduct undergo capacitation, including motility hyperactivation, as the time of ovulation approaches. Capacitating sperm shed proteins that bind them to the mucosal epithelium, while hyperactivation assists the sperm in pulling away from the epithelium and escaping out of mucosal pockets. The process of sperm release is gradual, reducing the chances of polyspermic fertilization that reduces embryo quality. Released sperm may be guided towards the oocyte by secretions of the oviduct, cumulus cells, or oocyte. Hyperactivation likely assists sperm in penetrating the cumulus matrix and is absolutely required for penetrating the zona pellucida and achieving fertilization. Mammalian oocytes take up only a small area of the lumen [42]. After fertilization in the ampulla, the cleaving embryo (shown in Figure 2E) develops for 5-7 days in vivo. In rats, mice, and humans, once the blastocyst reaches the uterine 
cavity, it orientates its embryonic pole towards the uterine epithelium and bursts out of the zona pellucida, a process known as hatching [44]. The epithelium is invaded from the lateral side of the blastocyst and the fragmented epithelial cells are phagocytosed by TE [44-46]. The blastocyst develops from a spherical to a tubular form and then elongates to a filamentous conceptus. In domestic ruminants (sheep, cattle, and goats), the elongation of the blastocyst marks the beginning of implantation, which involves apposition, followed by transient attachment and finally firm adhesion [47]. On the basis of these physiological events in the female reproductive tract, we aim to improve RT by mimicking the dynamic environment in vitro through development of elastomer-based microfluidic devices.

\subsection{Microfluidic channels to handle sperm and embryos}

Microfluidic devices which come into contact with reproductive cells can be fabricated using mechanical drilling or molding. Mechanical drilling is feasible for rapid prototyping because of the small volume to be removed; however, tool marks in the fabricated microfluidic channel caused by the drilling are undesirable for use in cell observation. For prototyping of the devices, molding methods using elastomers to create plastic devices are based on replication and are faster than those used on glass and harder plastics [48]. A combination of molding and use of elastomers is applicable to RT to mimic the physiological environment. The hydrophobic silicone elastomer PDMS, having a contact angle of $110^{\circ}$, is a key material capable of extending device applications for RT because it is nontoxic, transparent, inexpensive, and easy to handle [9]. The Young's modulus of PDMS is $100 \mathrm{kPa}$ [49]. The softness of the material enables easy fabrication, soft mechanism, and appropriate combinations with actuation systems. We classified the characteristics of the material and found it to be hydrophobic, transparent, elastic, and easy to pattern. PDMS microfluidic devices prepared by molding the microstructure and bonding the cured structure with a cover glass or glass slide can be used for manipulation and culture of cells to investigate their physiological functions. Within the last decade, studies using PDMS microfluidic channels or funnels have suggested novel solutions for oocyte manipulation, sperm sorting, and embryo culture [50-74]. Microfluidic systems that mimic oviductal structures and functions for use in RT are divided into those used for sperm motility control or monitoring [50-60], regulation of chemical gradients for in vitro fertilization and embryo culture [61-69], and applying mechanical stimuli to the developing embryo [70-74]. Although the unique characteristics of elastomers have been exploited for microstructure fabrication and/or micro-pumping, mechanical deformation of the elastomer membrane has not been used to produce new devices for RT. Applications developed by our group based on this elastomeric characteristic are introduced in this chapter.

\section{Sorting and analyzing motile sperm}

\subsection{Elastomer chip device for sperm sorting}

Ejaculated motile sperm is selected for successful fertilization in the oviduct. In ART, selection and sorting of motile sperm are routine processes. Some procedures may take up to $2 \mathrm{~h}$ for semen processing by conventional protocols, such as density gradient centrifugation and 
subsequent swim-up [75]. To reduce treatment times and physical damage induced by centrifugation, microfluidic sperm sorting (MFSS) chip devices have been developed for selecting motile sperm for use in ART [50-55]. As shown in Figure 3A, two gravity-driven laminar flows within the microfluidic channel are important for sperm selection. The fluids flowing through the semen inlet (A) and the medium inlet (B) should move parallel to each other and then exit through their respective outlets $(A \rightarrow D$ and $B \rightarrow C)$. Sperm are sorted on the basis of their ability to swim across the streamline into the medium stream, and hence only motile sperm are recovered in outlet C. Using an MFSS device, embryologists can perform a 1step sorting protocol without centrifugation and complete processing within $30 \mathrm{~min}$ [76]. Reducing the treatment time and eliminating the centrifugation step minimizes the exposure of sperm to concentrated reactive oxygen species (ROS) and prevents DNA fragmentation [77]. Schulte et al. previously reported that DNA fragmentation was significantly decreased in MFSS-treated sperm [53]. Technologies that facilitate the identification and selection of sperm with high motility should increase the ART success rate, and the linear velocities (LV) of sperm separated using an MFSS device have been shown to increase compared with those of untreated sperm [54,55]. On the basis of these results, an MFSS device can be used in clinical semen processing protocols for efficient ICSI and IVF. In the first report, the device was fabricated using PDMS bonded to a glass slide as shown in Figure 3B. Currently, disposable plastic devices for human ART are commercially available, as shown in Figure 3C. This device is a successful example of facilitated prototyping using elastomers.
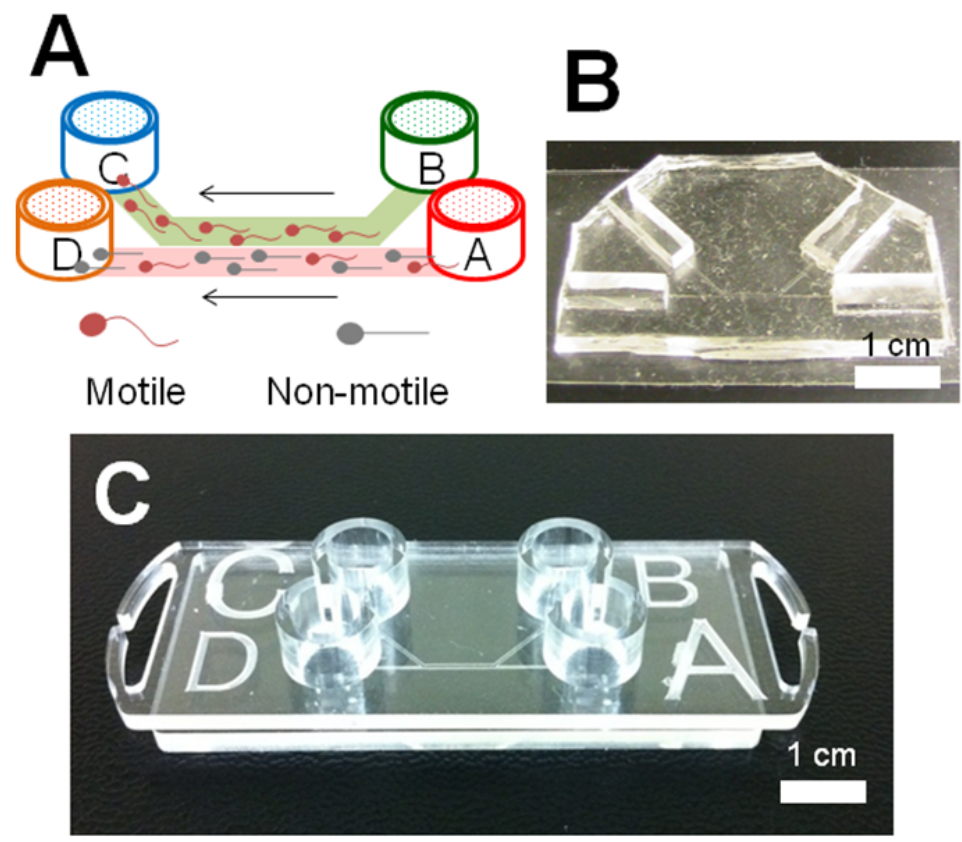

Figure 3. (A) Principle of sperm sorting using an MFSS device. Arrows show the direction of the laminar flow. (B) A glass-bottomed PDMS-MFSS. (C) A cycloolefin polymer-based MFSS device for human ART (Menicon Co., Ltd.). 


\subsection{Elastomer chip device for analyzing motility of motile sperm}

Sperm tracking and motility analyses are usually performed before and after sorting in ART. Optical microscopes have been used to image sperm for CASA and for manual identification of sperm motility for ART. A minimal image platform is desired to enable compactness, ease-of-use, minimized footprint, and portability to monitor sperm motility by a preclinical assay at home. Some researchers have developed a microfluidic chip that can be used by an individual himself at home on convenient moments to check his semen quality [56-59]. For example, if a person finds that his semen quality is low or that there are no motile sperm in his semen, he can opt for ICSI for his fertility treatment. McCormack et al. developed a fluorescent labeling method for simultaneously analyzing motile sperm concentration and motility by monitoring fluorescent intensity of a region where motile sperm were collected [56]. After a certain time period, the intensity of fluorescence was measured to quantify motile sperm concentration. Both motile sperm concentration and motility were taken into account by this design. However, fluorescent labeling might not be suitable for routine use in hospitals or at home. The microdevice produces a flow field for sperm to swim against, and sperm that overcome the flow within a specified time are propelled along in a separate channel and counted by the resistive pulse technique [57]. The microdevice could be used for simple self-assessment of sperm quality at home. Recently, an on-chip device for concentration of sperms using electrical impedance measurements was developed by Segerink et al. [58]. Using this device, the concentration of sperm and other cells, such as HL60 cells, in suspension can be determined. A lensless charge-coupled device (CCD) was integrated with a microfluidic chip to create a wide field of view combined with automatic recording as the sperm move inside a microfluidic channel [59]. These devices can evaluate sperm concentration and motility and the microfluidic channels can be fabricated using glass or poly(methyl methacrylate) (PMMA). However, for effective working of these devices, the physical characteristics of silicone elastomer such as hydrophobicity and elasticity are not required.

\subsection{Elastomeric chambers for recording trajectories of motile sperm}

Sperm motility analysis is not only used in human ART, but is also one of the key procedures used to analyze cattle and porcine sperm, especially frozen or transferred sperm used for AI. As discussed above, diluted semen is usually sandwiched between hydrophilic glass slides or glass- or plastic-bottomed microfluidic channels for observation of motile sperm. The trajectories of human and bovine sperm can be recorded using glass equipment; however, it is difficult to record the trajectory of motile porcine sperm using such a device because they adsorb to the surface of glass and hydrophilic plastics such as PMMA. Using a hydrophobic PDMS preparation (as shown in Figure 5), it would be possible to record the trajectories of motile sperm without problems associated with adsorption, making it possible to compare sperm motility parameters [60]. Because of the elasticity of PDMS, we propose using a preparation device such as that shown in Figure 5 for conventional CASA 
to reduce the overlap of motile sperm images (Figure 5D), which are artifacts of CASA. This device could thus be used to check for the quality of adhesive motile sperm and to investigate porcine sperm motility and AI success rate. The quality of porcine semen affects the nutrition or transfer of semen. Before AI, a technician needs to check the sperm quality. A device such as this could also be used to record adhesive human motile sperm adsorbed to glass in human ART.
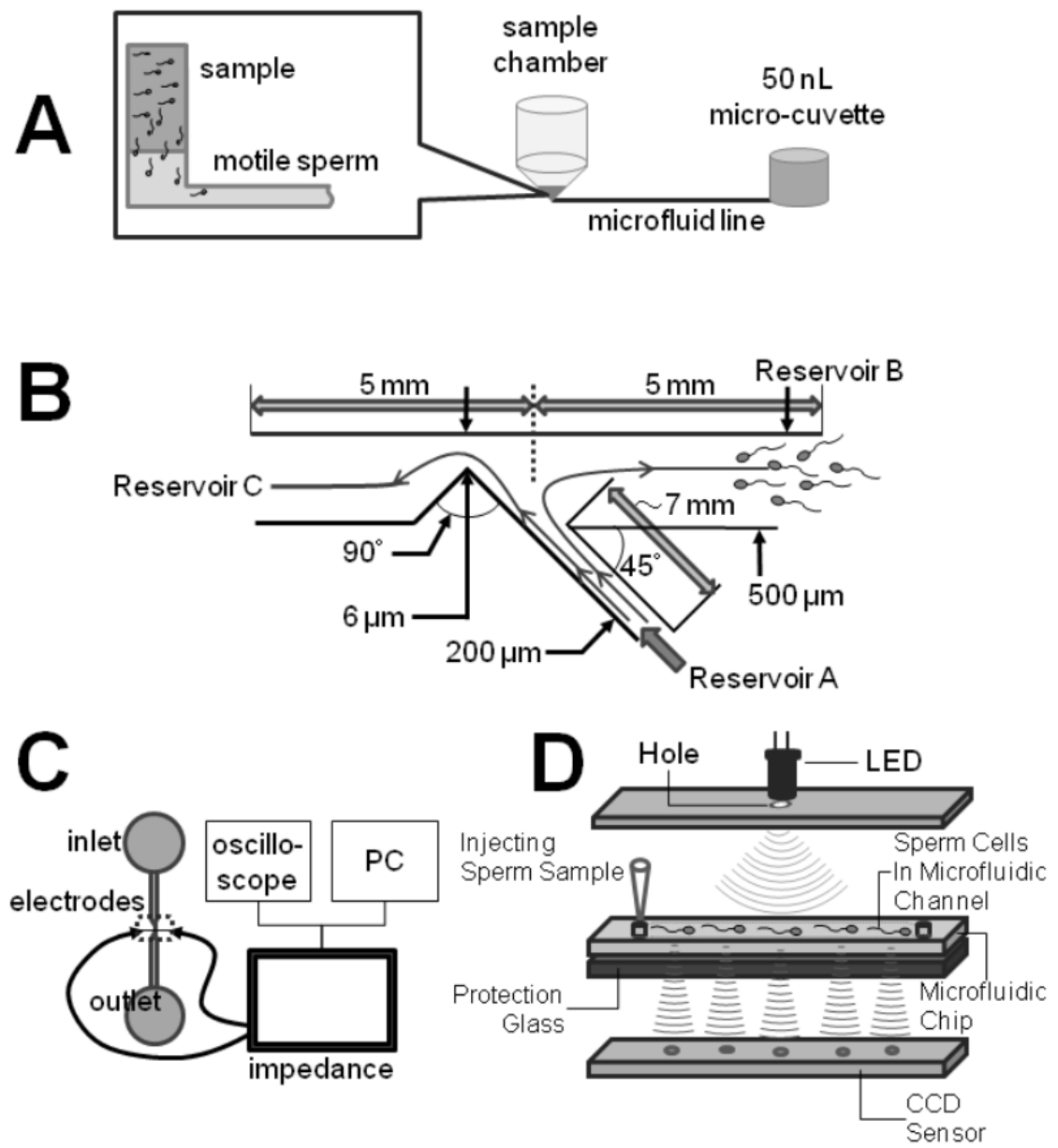

Figure 4. Sperm-analyzing microfluidic devices (A) A microfluidic device for monitoring fluorescent intensity of motile sperm through a microfluid line in the $50 \mathrm{~nL}$ micro-cuvette [56]. (B) A home-use device that allows rapid and quantitative sperm quality analysis using voltage measurements [57]. After a set time period, sperm arrive at the junction and the sperms swimming against the flow from $A$ to $B$ are flushed out through the aperture to reservoir $C$ by the fluid flow from A to C. (C) On-chip device for sperm concentration using electrical impedance measurements [58]. (D) A lensless charge-coupled device (CCD) integrated with a microfluidic chip [59]. 

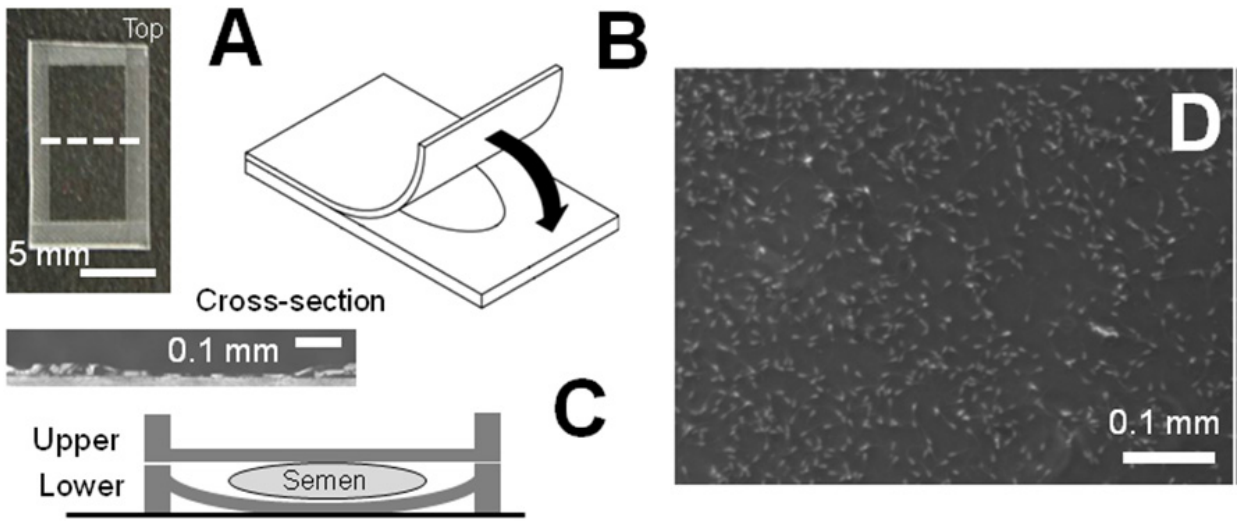

Figure 5. Use of a PDMS membrane for sperm motility analysis. (A) A PDMS membrane before use, with an area of $0.5 \times 1 \mathrm{~mm}^{2}$. (B) Method of sandwiching semen between the two membranes with a thickness of $0.1 \mathrm{~mm}$. (C) Cross-sectional image of the preparation. A water droplet is sandwiched between two PDMS membranes. (D) Sperm in this preparation is displayed by CASA. No overlap of motile sperm is observed in this frame.

\section{Static and dynamic mammalian embryo culture systems}

\subsection{Embryo culture systems using PDMS chambers or channels}

Fertilized oocytes can be cultured in several microliters of optimized medium for culture for longer than 5 days. In various animal models, increased embryo density has been shown to improve development, possibly through secretion of autocrine/paracrine factors. These growth factors may influence embryo development [78-80]. Based largely on data from animal models, several novel culture approaches utilizing reduced volumes to culture embryos appear to offer potential benefits for subsequent embryo development [79, 80]. However, using a low volume of medium may render the embryos susceptible to detrimental changes in conditions such as osmolality or $\mathrm{pH}$ [81]. To control beneficial and detrimental chemical environments during in vitro embryo culture, various systems including PDMS-based microfluidic devices have been developed. These are known as microwells or microfluidic channels.

The well-of-the-well system (WOW) has been used successfully with embryos from a variety of species including mouse, pig, cow, and human and entails using small impressions, or microwells, of varying sizes and arrangements created in the bottom of a vessel/dish $[8,61]$ (Figure 6A). An alternate approach that permits a commercial means of utilizing microwells in conjunction with existing dishware in the lab involves using microwell-inserts consisting of several rows of tiny culture wells composed of PDMS [38]. Within PDMS and glass-bottomed microchannels under static media conditions, 2-cell mouse embryos can be cultured to the blastocyst stage [65, 66]. However, researchers found that culture in microchannels resulted in significantly greater blastocyst formation and hatched blastocyst development at $72 \mathrm{~h}$ and 96 $\mathrm{h}$, respectively. These static culture systems could therefore be useful in investigating the impact of autocrine/paracrine compounds versus embryo spacing. 
A weak point of the use of PDMS for embryo development was evaporation of the medium through the PDMS due to the porous structure of the cured devices. This evaporation through the PDMS is significant and could result in marked shifts in medium osmolality and a resulting reduction in embryo quality. This drawback of PDMS was circumvented by design and use of a sandwich membrane that remained flexible, yet protected against evaporation [70]. A nonporous PDMS microwell culture system supported the developmental competence of bovine and human embryos cultured individually [64]. An important process in the fabrication of nonporous PDMS microwells is curing under low pressure $(-0.08 \mathrm{mPa})$. As a result, reports suggest that evaporation and osmolality changes can be reduced by appropriate fabrication techniques $[64,70]$.

\subsection{Elastomer channels and devices for dynamic embryo culture systems}

In vivo the preimplantation embryo is continually moving due to muscle contractions and movement of epithelial cell cilia [80]. This movement also disrupts cell-surface gradients that can form around embryos in static culture. It has been experimentally demonstrated that gradients exist in culture medium under static culture conditions because of embryo secretions or depletion of medium components. Gradients of potassium, calcium, and oxygen have been measured around mouse embryos $[82,83]$ and dynamic culture systems (DCSs) may disrupt these gradients, providing a more homogenous environment that more closely recapitulates the in vivo growth environment. These cilia and oviduct movements (shown in Figures 2A and B) also result in mechanical effects such as shear stress (SS), compression, and friction in the fallopian tube, which may influence developing embryos before nidation. DCSs may apply MS to the cultured embryo and thus replicate the environment it experiences under physiological conditions.

When using DCSs, there is a suggested threshold of SS that causes detrimental effects, such as apoptosis, on the cultured embryo. Xie et al. reported that SS in excess of $1.2 \mathrm{dyn} / \mathrm{cm}^{2} \mathrm{can}$ cause damage to blastomeres, up-regulation of stress signaling pathway constituents, and embryo degeneration [84]. Therefore, the SS in the vicinity of a cultured embryo induced by motions of DCSs should be kept below the threshold. In a study using a microfunnel as a DCS (Figure 6B), embryos were placed into the funnel reservoir, while medium was added and removed via a microfluidic channel connected to the bottom of the funnel via actions of a Braille actuator [71]. Mouse embryos were cultured in microdrop-static control, microfunnel-static control, or microfunnel-dynamic conditions with microfluidics. Progression to the blastocyst developmental stage was significantly enhanced under dynamic microfunnel culture conditions, as evidenced by an increased percentage of hatching or hatched blastocysts and a significantly higher average number of cells per blastocyst. Kim et al. developed a DCS using a combination of a PDMS microfluidic channel and a tilting machine, as shown in Figure 6C [72]. Bovine embryos were loaded and incubated by simply placing them on a tilting machine to provide embryo movement via gravity. The proportion of embryos that progressed to the eight-cell stage was superior in the constricted channel to that in the straight channel. Positive effects using tilting culture systems were also found in a microdroplet environment. We developed a tilting embryo 
culture system (TECS) to move mouse, porcine, and human embryos by fluid motion of the microdroplet, and we have shown that blastocyst development rates of these embryos using TECS improved over those of static controls [85-87]. Gentle vibration of porcine and human embryos also appeared to yield improved blastocyst formation rates [88-90]. These DCSs can be combined with microwells and microfluidic channels for mammalian embryo cultures.

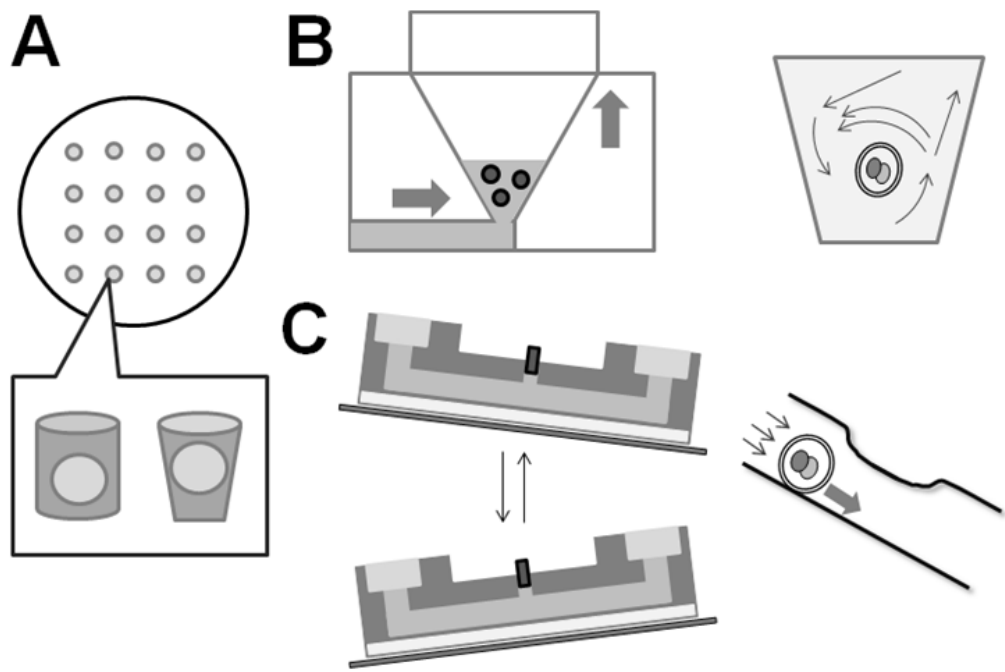

Figure 6. PDMS-based microfluidic devices for embryo culture. (A) The WOW system, (B) A microfunnel (C) A microfluidic IVC channel. In (B) and (C), the left and right figures show reservoir or microfluidic channels of the system and embryo and fluid motions, respectively.

\subsection{Peristaltic movement of an elastomer membrane to create a dynamic embryo culture system}

MS may be generated by fluid dynamics and compression of embryos due to interactions with the wall of the oviduct. We developed an air actuating system with microfluidic channels to apply MS by deforming a 0.1-mm-thick PDMS membrane and evaluated the MS applied to mouse embryos inside the microfluidic channel. Using an air actuating system as shown in Figures 7A and B, we applied compression to mouse embryos inside the medium channel and estimated SS based on the velocity of the embryos' motion [74, 75]. It has been demonstrated that this culture system can be employed to investigate the relationship between MS and molecular mechanisms. Because this culture system could be directly positioned on the stage of a fluorescence microscope, fluorescence images of embryos in the microfluidic channel could be recorded, indicating the distribution of intracellular calcium concentration $\left(\left[\mathrm{Ca}^{2+}\right]_{i}\right)$. Both MS and $\left[\mathrm{Ca}^{2+}\right]_{i}$ were quantified based on time-resolved confocal microscopy images. When blastocysts were compressed, FI increased in response to the applied MS, as shown in Figure 7C. Molecular mechanosensing systems such as mechanosensitive ion channels could play an important role in responses to these MS. Using this device to investigate the applied MS and resultant molecular response, we can investigate the functions 
of the embryonic sensor proteins. The ultimate goal is to use this mechanism as an artificial oviduct for clinical use based on the soft mechanism of elastomers.

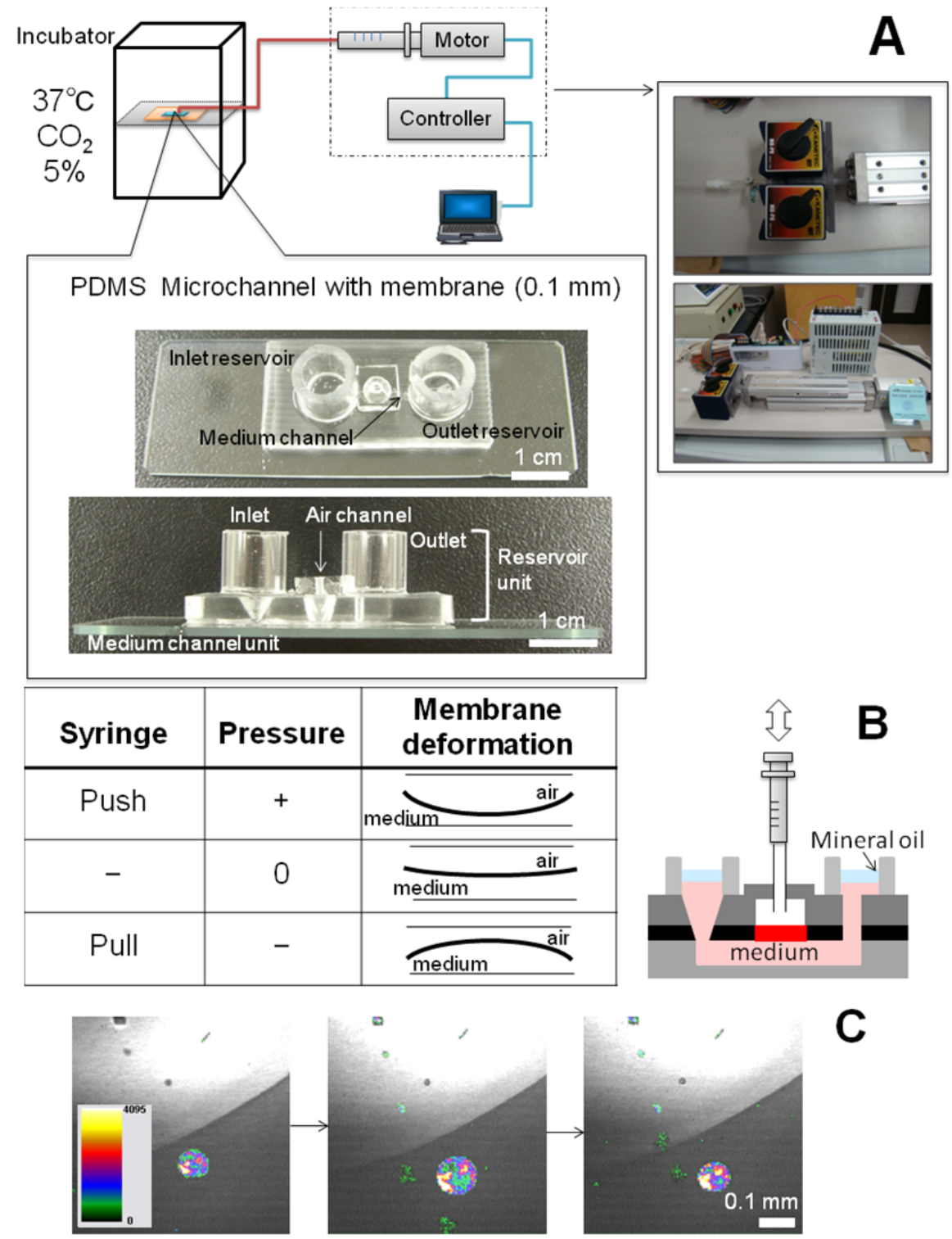

Figure 7. A pneumatic microfluidic actuation system for dynamic embryo culture. (A) Mechanical drive system and glass-bottomed PDMS microfluidic channel. (B) Schematic view of PDMS membrane deformation. The thick line represents the PDMS membrane. (C) $\left[\mathrm{Ca}^{2+}\right]_{i}$ changes in a compressed mouse blastocyst in the microfluidic channel induced by membrane deformation. The center image shows embryo compression. 


\section{Conclusion}

We developed microfluidic devices and systems for use in motile sperm sorting, sperm motility checking, and static and dynamic embryo culture systems based on the physical and chemical properties of silicone elastomers. Utilizing these properties, methods for RT can be improved, and novel strategies can be developed to implement more physiological treatments for human ART. However, most commercial elastomers are not recommended for medical use. To apply microfluidic channels in dynamic human embryo culture for ART, it is necessary to select a soft material approved for clinical use to prepare the microfluidic channels. Silicone hydrogel, which is used in the manufacture of contact lenses, is a candidate material for this application; therefore, we developed silicone hydrogel microfluidic channels for embryo culture. Once commercial elastomers have been approved for clinical use, elastomer-based medical microdevices are likely to become widespread and routinely used. The material development and approval of the developed materials for medical use are bottlenecks for the medical application of elastomeric devices and systems. This problem also applies to implantation and regenerative medicine. Over the last decade, great progress has been made in RT and human ART, and these improvements can contribute to other fields of medicine utilizing microfluidic systems.

\section{Author details}

Koji Matsuura*

Research Core for Interdisciplinary Sciences, Okayama University, Okayama, Japan

Keiji Naruse

Cardiovascular Physiology, Graduate School of Medicine, Dentistry and Pharmaceutical Sciences, Okayama University, Okayama, Japan

\section{Acknowledgement}

This study was partly supported by a grant-in-aid for Scientific Research for Young Scientists (B) and (A), and Challenging Exploratory Research (Nos. 20700380, 22680036, 23650262 to K. M.) and Special Coordination Funds for Promoting Sciences and Technology from the Ministry of Education Science Sports and Culture, Japan (K. M.). K. M. thanks to Mieko Kodama (Okayama University) for her assistance with figure preparation and formatting. The authors would like to thank Enago for the English language review.

\section{References}

[1] Okere C, Nelson L (2002) Novel Reproductive Techniques in Swine Production-A Review. Asian-Aust. J. Anim. Sci. 15: 445-452.

[2] William VH, Amanda RP (1999) Role of reproductive technologies and genetic resource banks in animal conservation. Rev. Reprod. 4: 143-150.

\footnotetext{
* Corresponding Author
} 
[3] Gardner DK (2006) In vitro fertilization-A practical approach. Informa health care

[4] Maes D, Nauwynck H, Rijsselaere T, Mateusen B, Vyt P, Kruif AD, Soom AV (2008) AI transmitted diseases in swine: an overview. Theriogenology. 70: 1337-1345.

[5] Vishwanath R (2003) Artificial insemination: the state of the art. Theriogeneology. 59: 571-584.

[6] Thibier M, Wagner HG (2000) World statistics for artificial insemination in cattle. Proc. 14th Int. Congr. Anim. Reprod. 22: 76.

[7] Moore K, Thatcher WW (2006) Major Advances Associated with Reproduction in Dairy Cattle. J. Dairy Sci. 89: 1254-1266.

[8] Vajta G, Peura TT, Holm P, Paldi A, Greve T, Trounson AO, Callesen H (2000) New method for culture of zona-included or zona-free embryos: the Well of the Well (WOW) system. Mol. Reprod. Dev. 55: 256-264.

[9] Douglas BW, George MW (2006) Applications of microfluidics in chemical biology. Cur. Opin. Chem. Biol. 10: 584-591.

[10] Velve-Casquillas G, Berre ML, Piel M, Tran PT (2010) Microfluidic tools for cell biological research. Nano Today. 5: 28-47.

[11] Holmes D, Pettigrew D, Reccius CH, Gwyer JD, Berkel CV, Holloway J, Davies DE, Morgan H (2009) Leukocyte analysis and differentiation using high speed microfluidic single cell impedance cytometry. Lab Chip. 9: 2881-2889.

[12] Wang J, Heo J, Hua SZ (2010) Spatially resolved shear distribution in microfluidic chip for studying force transduction mechanisms in cells. Lab Chip. 10: 235-239.

[13] Tan JL, Tien J, Pirone DM, Gray DS, Bhadriraju K, Chen CS (2003) Cells lying on a bed of microneedles: An approach to isolate mechanical force. Proc. Natl. Acad. Sci. U.S.A. 100: $1484-1489$.

[14] Roure OD, Saez A, Buguin A, Austin RH, Chavrier P, Silberzan P, Ladoux B (2005) Force mapping in epithelial cell migration. Proc. Natl. Acad. Sci. U.S.A. 102: 2390-2395.

[15] Vanapalli SA, Duits MHG, Mugele F (2009) Microfluidics as a functional tool for cell mechanics. Biomicrofluidics. 3: 012006.

[16] Chiu DT, Jeon NL, Huang S, Kane RS, Wargo CJ, Choi IS, Ingber DE, Whitesides GM (2000) Patterned deposition of cells and proteins onto surfaces by using threedimensional microfluidic systems. Proc. Natl. Acad. Sci. U.S.A. 97: 2408-2413.

[17] Hung PJ, Lee PJ, Sabounchi P, Lin R, Lee LP (2005) Continuous perfusion microfluidic cell culture array for high-throughput cell-based assays. Biotechnol. Bioeng. 89: 1-8.

[18] Koyama S, Amarie D, Soini HA, Novotny MV, Jacobson SC (2006) Chemotaxis assays of mouse sperm on microfluidic devices. Anal. Chem. 78: 3354-3359.

[19] Shamloo A, Ma N, Poo M, Sohn LL, Heilshorn SC (2008) Endothelial cell polarization and chemotaxis in a microfluidic device. Lab Chip. 8: 1292-1299.

[20] Mapletoft RJ, Hasler JF (2005) Assisted reproductive technologies in cattle: a review. Rev. sci. tech. Off. int. Epiz. 24: 393-403.

[21] Chan WS, Dixon ME (2008) The "RT" of thromboembolism : A review of assisted reproductive technology and thromboembolic complications. Thromb. Res. 121: 713726. 
[22] Kim LU, Johnson MR, Barton S, Nelson MR, Sontag G, Smith JR, Gotch FM, Gilmour JW (1999) Evaluation of sperm washing as a potential method of reducing HIV transmission in HIV-discordant couples wishing to have children. AIDS. 13: 645-651.

[23] Aitken RJ, Clarkson JS (1988) Significance of reactive oxygen species and antioxidants in defining the efficacy of sperm preparation techniques. J. Androl. 9: 367-376.

[24] Alvarez1 JG, Lasso JL, Blasco L, Nuñez RC, Heyner S, Caballero PP, Storey BT (1993) Centrifugation of human spermatozoa induces sublethal damage; separation of human spermatozoa from seminal plasma by a dextran swim-up procedure without centrifugation extends their motile lifetime. Hum. Reprod. 8: 1087-1092.

[25] Sharma RK, Vemulapalli S, Kohn S, Agarwal A (1997) Effect of Centrifuge Speed, Refrigeration Medium, and Sperm Washing Medium on Cryopreserved Sperm Quality After Thawing. Sys. Biol. Reprod. Med. 39: 33-38.

[26] Hirano Y, Shibahara H, Obara H, Suzuki T, Takamizawa S, Yamaguchi C, Tsunoda H, Sato I (2001) Relationships Between Sperm Motility Characteristics Assessed by the Computer-Aided Sperm Analysis (CASA) and Fertilization Rates In Vitro. J. Assist. Reprod. Genet. 18: 215-220.

[27] Agarwal A, Sharma RK (2007) Automation is the key to standardized semen analysis using the automated SQA-V sperm quality analyzer. Fertil. Steril. 87: 156-162.

[28] Zhang BR, Larsson B, Lundeheim N, Rodriguez-Martinez H (1998) Sperm characteristics and zona pellucida binding in relation to field fertility of frozen-thawed semen from dairy AI bulls. Int. J. Androl. 21: 207-216.

[29] England GCW (1992) The Cryopreservation of Canine Semen. Thesis, University of London, UK.

[30] Rotaa A, Penã AI, Linde-Forsberg C, Rodriguez-Martinez H (1999) In vitro capacitation of fresh, chilled and frozen-thawed dog spermatozoa assessed by the chlortetracycline assay and changes in motility. Anim. Reprod. Sci. 57: 199-215.

[31] Holzer HE, Chain RC, Demirtas E, Ba-Akdah H, Tan SL (2006) In vitro maturation of oocytes. In: Gardner DK editor, In vitro fertilization-A practical approach. Informa health care. pp. 127-145.

[32] Galli C, Lazzari G (1996) Practical aspects of IVM/IVF in cattle. Anim. Reprod. Sci. 42: 371-379.

[33] Hoover L, Baker A, Check JH, Lurie D, Summers D (1997) Clinical outcome of cryopreserved human pronuclear stage embryos resulting from intracytoplasmic sperm injection. Fertil. Steril. 67: 621-624.

[34] Devroey P, Steirteghem AV (2004) A review of ten years experience of ICSI. Hum. Reprod. Update. 10: 19-28.

[35] Keskintepe L, Pacholczyk G, Machnicka A, Norris K, Curuk MA, Khan I, Brackett BG (2002) Bovine blastocyst development from oocytes injected with freezedried spermatozoa. Biol. Reprod. 67: 409-415.

[36] Ebner T (2006) Embryo development and assessment of viability. In: Gardner DK, editor. In vitro fertilization-A practical approach. Informa health care, pp 199-220.

[37] Gardner DK, Lane M, Spitzer A, Batt PA (1994) Enhanced rates of cleavage and development for sheep zygotes cultured to the blastocyst stage in vitro in the absent of 
serum and somatic cells-amino acids, vitamins, and cuhuring embryos in groups stimulate development. Biol. Reprod. 50: 390-400.

[38] Mapletoft RJ (2006) Bovine Embryo Transfer, IVIS Reviews in Veterinary Medicine, I.V.I.S. (Ed.). International Veterinary Information Service, Ithaca NY

[39] Strandell A, Bergh C, Lundin K (2000) Selection of patients suitable for one-embryo transfer reduces the rate of multiple births by half without impairment of overall birth rates. Hum. Reprod. 15: 2520-2525.

[40] Bergh C (2005) Single embryo transfer: a mini-review. Hum. Reprod. 20: 323-327.

[41] Gandolfi F (1994) Autocrine, paractine and environmental factors influencing embryonic development from zygote to blastocyst. Theriogenology. 41: 95-100.

[42] Suarez SS (2008) Regulation of sperm storage and movement in the mammalian oviduct, Int. J. Dev. Biol. 52: 455-462.

[43] Suarez SS (2008) Control of hyperactivation in sperm, Hum. Reprod. Update. 14: 647657.

[44] Yoshinaga K (2010) Research on Blastocyst Implantation Essential Factors (BIEFs). Am. J. Reprod. Immun. 63: 413-424.

[45] Enders AC, Schlafke S (1967) A morphological analysis of the early implantation stages in the rat. Am. J. Anat. 120: 185-226.

[46] Bischof1 P, Campana A (1996) A model for implantation of the human blastocyst and early placentation. Hum. Reprod. Update. 2: 262-270.

[47] Spencer TE, Johnson GA, Bazer FW, Burghardt RC (2004) Implantation mechanisms: insights from the sheep. Reproduction. 128: 657-668.

[48] Duffy DC, McDonald JC, Schueller OJA, Whitesides GM (1998) Rapid Prototyping of Microfluidic Systems in Poly(dimethylsiloxane). Anal. Chem. 70: 4974-4984.

[49] Gray DS, Tien J, Chen CS (2003) Repositioning of cells by mechanotaxis on surfaces with micropatterned Young's modulus. J. Biomed. Mater. Res. 66A: 605-614.

[50] Cho BS, Schuster TG, Zhu X, Chang D, Smith GD, Takayama S (2003) Passively Driven Integrated Microfluidic System for Separation of Motile Sperm. Anal. Chem. 75: 16711675.

[51] Schuster TG, Cho B, Keller LM, Takayama S, Smith GD (2003) Isolation of motile spermatozoa from semen samples using microfluidics. Reprod. Biomed. Online. 7: 7581.

[52] Wu JM, Chung Y, Belford KJ, Smith GD, Takayama S, Lahann J (2006) A surfacemodified sperm sorting device with long-term stability. Biomed. Microdev. 8: 99-107.

[53] Schulte RT, Chung YK, Ohl DA, Takayama S, Smith GD (2007) Microfluidic sperm sorting device provides a novel method for selecting motile sperm with higher DNA integrity. Fertil. Steril. 88: S76.

[54] Shibata D, Ando H, Iwase A, Harata T, Kikkawa F, Naruse K (2007) Analysis of sperm motility and fertilization rates after the separation by microfluidic sperm sorter made of quartz. Fertil. Steril. 88: S110.

[55] Matsuura K, Takenami M, Kuroda Y, Hyakutake T, Yanase S, Naruse K (2012) Screening of Sperm Velocity by Fluid Mechanical Characteristics of a Cycloolefin Polymer Microfluidic Sperm Sorting Device. Reprod. Biomed. Online. 24: 109-115. 
[56] McCormack MC, McCallum S, Behr B (2006) A novel microfluidic device for male subfertility screening. J. Urol. 175: 2223-2227.

[57] Chen YA, Huang ZW, Tsai FS, Chen CY, Lin CM, Wo AM (2011) Analysis of sperm concentration and motility in a microfluidic device. Microfluid. Nanofluid. 10: 59-67.

[58] Segerink LI, Sprenkels AJ, Braak PMT, Vermes I, Berg AVD (2011) On-chip determination of spermatozoa concentration using electrical impedance measurements. Lab Chip. 10: 1018-1024.

[59] Zhang X, Khimji I, Gurkan UA, Safaee H, Catalano PN, Keles HO, Kayaalp E, Demirci U (2011) Lensless imaging for simultaneous microfluidic sperm monitoring and sorting. Lab Chip. 11: 2535-2540.

[60] Matsuura K, Kuroda Y, Yamashita K, Funahashi H (2011) Hydrophobic Silicone Elastmer Chamber for Recording Trajectories of Porcine Motile Sperms without Adsorption. J. Reprod. Dev. 57: 163-167.

[61] Vajta G, Korosi T, Du Y, Nakata K, Ieda S, Kuwayama M, Nagy ZP (2008) The Well-ofthe-Well system: an efficient approach to improve embryo development. Reprod. Biomed. Online. 17: 73-81.

[62] Sugimura S, Akai T, Somfai T, Hirayama M, Aikawa Y, Ohtake M, Hattori H, Kobayashi S, Hashiyada Y, Konishi K, Imai K (2010) Time-lapse cinematographycompatible polystyrene-based microwell culture system: a novel tool for tracking the development of individual bovine embryos. Biol. Reprod. 83: 970-978.

[63] Cheng CM, Matsuura K, Wang IJ, Kuroda Y, Leduc PR, Naruse K (2009) Fabricating small-scale, curved, polymeric structures with convex and concave menisci through interfacial free energy equilibrium. Lab Chip. 9: 3306-3309.

[64] Hashimoto S, Kato N, Saeki K, Morimoto Y, (2012) Selection of high-potential embryos by culture in poly(dimethylsiloxane) microwells and time-lapse imaging. Fertil Steril. 97: 332-337.

[65] Raty S, Davis J, Beebe D, Rodriguez-Zas S, Wheeler M (2001) Culture in microchannels enhances in vitro embryonic development of preimplantation mouse embryos. Theriogenology. 55: 241.

[66] Raty S, Walters EM, Davis J, Zeringue H, Beebe DJ, Rodriguez-Zas SL, Wheeler MB (2004) Embryonic development in the mouse is enhanced via microchannel culture. Lab Chip. 4: 186-190.

[67] Zeringue HC, Rutledge JJ, Beebe DJ (2005) Early mammalian embryo development depends on cumulus removal technique. Lab Chip. 5: 86-90.

[68] Beebe D, Wheeler M, Zeringue H, Walters E, Raty S (2002) Microfluidic technology for assisted reproduction. Theriogenology. 57: 125-135.

[69] Krisher RL, Wheeler MB (2010) Towards the use of microfluidics for individual embryo culture. Reprod. Fertil. Dev. 22: 32-39.

[70] Heo YS, Cabrera LM, Song JW, Futai N, Tung YC, Smith GD, Takayama S (2007) Characterization and resolution of evaporation-mediated osmolality shifts that constrain microfluidic cell culture in poly(dimethylsiloxane) devices. Anal. Chem. 79: 1126-1134. 
[71] Heo YS, Cabrera LM, Bormann CL, Shah CT, Takayama S, Smith GD (2010) Dynamic microfunnel culture enhances mouse embryo development and pregnancy rates. Hum. Reprod. 25: 613-622.

[72] Kim MS, Bae CY, Wee G, Han YM, Park JK (2009) A microfluidic in vitro cultivation system for mechanical stimulation of bovine embryos. Electrophoresis. 30: 3276-3282.

[73] Li JC, Matsuura K, Kuroda Y, Funahashi H, Naruse K (2010) Application of mechanical stimuli using a microfluidic air actuating system to cultured mammalian embryos. MHS 2010 abstract: 29-34.

[74] Matsuura K, Watanabe K, Kodama M, Kuroda Y, Naruse K (2011) Development of observation system to investigate both intracellular calcium concentration and mechanical stimuli to mammalian embryos. MHS 2011 abstract: 99-104.

[75] Gautam N, Allahbadia R, Basuray D, Rubina M (2003) The Art and Science of Assisted Reproductive Techniques. Taylor \& Francis: 213-221. Chapter 30.

[76] Hughes CM, Lewis SE, McKelvey-Martin VJ, Thompson W (1998) The effects of antioxidant supplementation during Percoll preparation on human sperm DNA integrity. Hum. Reprod. 13: 1240-1247.

[77] Mortimer D (1991) Sperm preparation techniques and iatrogenic failures of in-vitro fertilization. Hum. Reprod. 6: 173-176.

[78] Richter KS (2008) The importance of growth factors for preimplantation embryo development and in-vitro culture. Curr. Opin. Obstet. Gynecol. 20: 292-304.

[79] Swain JE, Smith GD (2011) Advances in embryo culture platforms: novel approaches to improve preimplantation embryo development through modifications of the microenvironment. Hum. Reprod. Update. 17: 541-557.

[80] Smith GD, Takayama S, Swain JE (2012) Rethinking In Vitro Embryo Culture: New Developments in Culture Platforms and Potential to Improve Assisted Reproductive Technologies. Biol. Reprod. 86: 62.

[81] Swain JE (2010) Environmental factors and manual manipulations during preparation influence embryo culture media osmolality. Fertil. Steril. 94(Suppl.): S32.

[82] Trimarchi JR, Liu L, Smith PJ, Keefe DL (2000) Noninvasive measurement of potassium efflux as an early indicator of cell death in mouse embryos. Biol. Reprod. 63: 851-857.

[83] Trimarchi JR, Liu L, Porterfield DM, Smith PJ, Keefe DL (2000) Oxidative phosphorylationdependent and -independent oxygen consumption by individual preimplantation mouse embryos. Biol. Reprod. 62: 1866-1874.

[84] Xie Y, Wang F, Zhong W, Puscheck E, Shen H, Rappolee DA (2006) Shear stress induces preimplantation embryo death that is delayed by the zona pellucida and associated with stressactivated protein kinase-mediated apoptosis. Biol. Reprod. 75: 45-55.

[85] Matsuura K, Hayashi N, Kuroda Y, Takiue C, Hirata R, Takenami M, Aoi Y, Yoshioka N, Habara T, Mukaida T, Naruse K (2010) Improved Development of Mouse and Human Embryos by Tilting Embryo Culture System. Reprod. BioMed. Online. 20: 358364.

[86] Koike T, Matsuura K, Naruse K, Funahashi H (2010) In-vitro Culture with a tilting device in chemically defined media during meiotic maturation and early development 
improves the quality of blastocysts derived from in-vitro matured and fertilized porcine oocytes. J. Reprod. Dev. 56: 552-557.

[87] Hara T, Naruse K, Matsuura K, Kodama T, Sato K, Tateaki Y (2010) A novel tilting embryo culture system (TECS) improves human blastocyst quality clinically. ESHRE2010.

[88] Mizobe Y, Yoshida M, Miyoshi K (2010) Enhancement of cytoplasmic maturation of in vitro-matured pig oocytes by mechanical vibration. J. Reprod. Dev. 56: 285-290.

[89] Isachenko E, Maettner R, Isachenko V, Roth S, Kreienberg R, Sterzik K (2010) Mechanical agitation during the in vitro culture of human pre-implantation embryos drastically increases the pregnancy rate. Clin. Lab. 56: 569-76.

[90] Isachenko V, Maettner R, Sterzik K, Strehler E, Kreinberg R, Hancke K, Roth S, Isachenko E (2011) In-vitro culture of human embryos with mechanical micro-vibration increases implantation rates. Reprod. BioMed. Online. 22: 536-544. 